\title{
CVD Growth of Mono- and Bi-Layer Graphene from Ethanol
}

\author{
Xiao Chen, Pei Zhao, Bo Hou, Shohei Chiashi and Shigeo Maruyama \\ Department of Mechanical Engineering, The University of Tokyo
}

\begin{abstract}
CVD on metal substrates has been provn effective in the synthesis of graphene. But before application in graphene electronics, the quality of the synthesized graphene needs to be improved. Therefore a reliable method to grow large-scale, highquality graphene is required. Besides, the mechanism of graphene growth is not fully understood. Here we report utilizing CVD to produce mono- and bi-layer graphene from ethanol. By using ethanol as carbon source, we carefully studied the CVD parameter for the growth process, and their effects on the defects and grain size of graphene. By changing growth condition we were able to efficiently control the layer number (1 or 2). We also employed ${ }^{12} \mathrm{C},{ }^{13} \mathrm{C}$, and ${ }^{13} \mathrm{CH}_{3} \mathrm{CH}_{2} \mathrm{OH}$ ethanol to synthesize graphene, and based on the spectroscopy results we are able to explain the growth mechanism on $\mathrm{Cu}$ and $\mathrm{Ni}$ substrates.
\end{abstract}

\section{INTRODUCTION}

Graphene, a monolayer of $\mathrm{sp}^{2}$-bonded carbon atoms or one monolayer of graphite, has triggered numerous fundamental and technological studies, due to its unique transport properties [1], which has motivated the development of largescale, high-quality graphene acquiring methods, such as mechanical exfoliation [2], graphitization of $\mathrm{SiC}$ surfaces [3], reduced graphene oxide [4] and chemical vapor deposition (CVD) on transition metals such as $\mathrm{Ni}$ [5] and $\mathrm{Cu}$ [6].

In this work we report our study on CVD growth of graphene on $\mathrm{Cu}$ and $\mathrm{Ni}$ substrates by using ethanol as carbon source. We found that compared with widely-used methane, ethanol is also capable of synthesizing high-quality, largescale graphene, but with higher safety and lower cost.

\section{Experiment}

We adopt commercially available $\mathrm{Cu}$ foils and thermally evaporated Ni films as substrates. We first clean the substrate surface by soaking it in $\mathrm{HCl}$ solution, acetone and 2-proponal, than we heat it to the CVD reaction temperature (typically, $900{ }^{\circ} \mathrm{C}$ for $\mathrm{Ni}$ and $1050{ }^{\circ} \mathrm{C}$ for $\mathrm{Cu}$ ) in a quartz tube. Afterwards, we anneal the substrate for 1 hour, so that largegrain and smooth surface could be formed (This step is not applied for $\mathrm{Ni}$ films). $3 \% \mathrm{H}_{2}$ in $\mathrm{Ar}$ is flowed in the reaction tube through the heating and annealing process. After annealing, we flow ethanol vapor into the tube. When CVD reaction finishes, we stop the ethanol flow and cool down the tube. The synthesized graphene is transferred with PMMA onto a $\mathrm{Si} / \mathrm{SiO}_{2}$ substrate by removing metal in etchant, before being further studied with SEM and Raman spectroscopy. We also conduct many experiments with different pressure, CVD
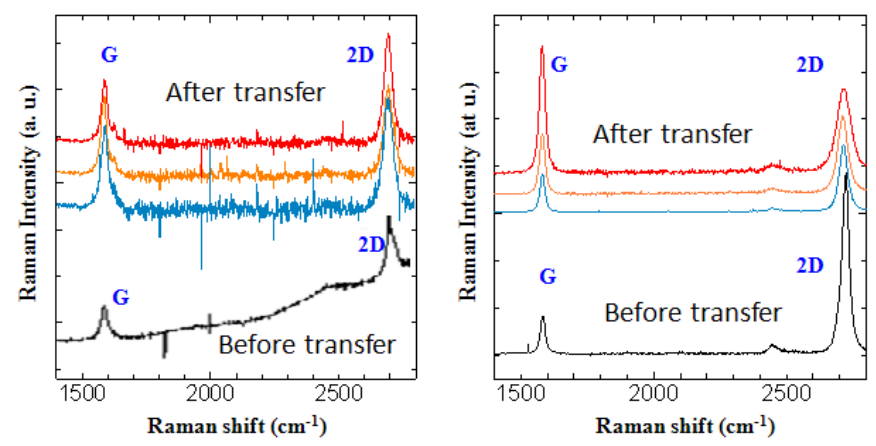

Fig. 1: Raman spectra of graphene grown (a) on $\mathrm{Cu}$ and (b)

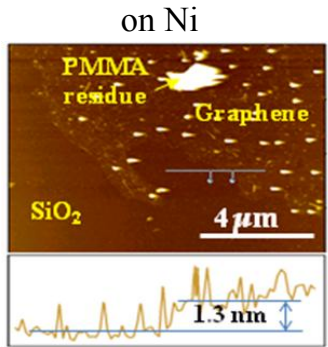

Fig. 2: AFM image of transferred graphene

time period and temperature, to examine the number of layers, defect level and grain size.

\section{Results}

The Raman spectra of graphene grown on $\mathrm{Cu}$ and $\mathrm{Ni}$ (Fig. 1) show that we have successfully synthesized mono- or bilayer graphene. The AFM image of graphene on $\mathrm{Si} / \mathrm{SiO}_{2}$ substrate helps to demonstrate the existence of graphene, due to the fact that the height difference at the cross section is 1.3 $\mathrm{nm}$. Based on our parametric studies along with ${ }^{12} \mathrm{C}$ and ${ }^{13} \mathrm{C}$ ethanol growth experiments, we provide a direct surface growth model for graphene growth on both $\mathrm{Cu}$ and $\mathrm{Ni}$.

This work was partially supported by the JSPS Core-toCore Program.

\section{REFERENCES}

[1] Geim, A. K.; Novoselov, K. S. Nat. Mater. 6 (2007) 183.

[2] K. S. Novoselov, A.K. Geim, et al.,Science 306 (2004) 666 .

[3] C. Berger, W. A.de Heer, et al., Science 312 (2006) 1191.

[4] S. Stankovic, R. S. Ruoff, et al., Carbon 45 (2007) 1558.

[5] K. S. Kim, et al., Nature 457 (2009) 706.

[6] X. Li, et al., Science 324 (2009) 1312. 